\title{
How Is Meaning Constructed Multimodally?-A Case Study of PowerPoint Presentations in an MA Thesis Defense
}

\author{
Zhencong Liu \\ School of English Language, Culture and Literature, Beijing International Studies University, China \\ Hui Liu \\ School of English Language, Culture and Literature, Beijing International Studies University, China
}

\begin{abstract}
Taking the theory of multimodal analysis as the theoretical basis and 7 ppts in an MA thesis defense as the research data, this paper uses a combination of qualitative and quantitative methods to analyze the distribution and realizations of types and modalities of PPTs in this MA thesis defense. The results show that the analyzing type accounts for the highest proportion to make sure the PPT defense presentation is academic and serious. The use of connecting, persuading, and prompting types has a strong personal tendency, varying from person to person. The visualizing type takes up the lowest proportion among them, indicating that pictures are the last modality that demonstrators tend to use. The paper further explores how meanings of MA thesis defense are constructed multimodally, and investigates the characteristics of PPT as a thesis defense genre, aiming to give some suggestions and instruction on ppt presentation for thesis defenses.
\end{abstract}

Index Term $s$-multimodality, ppt presentation, MA thesis defense, genre

\section{INTRODUCTION}

In today's era, the ways of communication are featured by timeliness, variety, multimodality. Though natural language remains the main method of communication, meanings are increasingly conveyed by other non-verbal semiotic resources, such as pictures, images, colors, sound and technologies, etc. (Kress \& Van, 2001). In this sense, PowerPoint presentation is an effective method of communication, realized by text, picture, chart, music, video, animation, etc., and it is featured by various multimodalities.

"PowerPoint itself has transcended mere software status to become a cultural icon of contemporary communication. More than 400 million copies of the program are currently in circulation, and somewhere between 20 and 30 million PowerPoint-based presentations are given around the globe each day" (Simons, 2005). In this respect, PPT, as a widely used presentation method, has become the dominant visual-aid medium, especially preferred by merchants, educators, and trainers, etc. While, many voices criticize PPT vehemently for its commercial use, the incoherence (Tuffe, 2003), obnoxious animation, lousy contrast, stupid sounds, and using and abusing phots (Altman, 2004).

According to $\mathrm{Hu}$ (2007), part of the reasons why there are two completely divergent opinions lies in the different understanding towards the concept of PPT. Yates and Orlikowski (2007, p.67-92) proposed that PPT can be defined from three perspectives, a tool of a presentation, a text of a presentation, and the genre of a presentation. PPT, as a tool, is software used to make intuitive content, such as images, animation, and audio, etc. It can express information to the audiences, while it cannot convey meaning. PPT, as a text, refers to the content on it, presented by different modalities, including visual, pictorial, audio forms. PPT, as a genre, is regarded as a socially recognized type of communicative action. Knoblauch (2007) clarifies the often-mixed meaning of PowerPoint and powerpoint. In his view, PowerPoint refers to the presentation software, usually being synonymous with Microsoft office. However, the latter refers to "a communicative action by a presenter, attended by an audience, involving technologies, such as a computer screen, a projector, or a slide, as well as activities performed in relation to both audiences and technologies". Knoblauch's view about powerpoint happens to coincide with Yates and Orlikowski's. The PPT presentation, as a genre, has become pervasive in various domains of communication. This paper will mainly adopt the notions of PPT as a text, more accurately a multimodal discourse, in the context of MA thesis defense, then an analysis will be given to explore the defense meaning constructed through different modalities, some improvement on the quality of PPT production. The characteristics of PPT as a thesis defense genre will also be discussed.

As an important part of graduate education, the thesis defense is the summative achievement of graduate education. It reflects the theoretical foundation, professional knowledge, academic level, and innovation ability of postgraduates. The thesis defense is also an important part of graduate education. Researches have been conducted to explore the skills (Zhou et al., 2008), conversation characteristics of thesis defense (Recski, 2005; Ke, 2010), and identity construction in 
thesis defense (Gu, 2017; Li \& Jing, 2019). However, none of them approach the perspective of PPT. Thus, this paper will focus on how the defense meaning is constructed multimodally, based on the PPT thesis defense genre.

\section{THEORETICAL BASIS}

\section{A. Modality and Multimodality}

Kress and Van Leeuwen (2001) propose that mode is the meaning potential formed by material media after long-term social shaping, and it is a social symbol resource used to represent and communicate meaning. Zhu (2007) believes that modality includes language, image, color, music, animation, and other symbolic systems, and it is the channel and medium of communication. Gu (2007) believes that modality refers to the interaction between human beings and the external environment through the sensory system (such as vision, hearing, etc.). Although the definition of modality is not completely unified, it is basically around a few keywords, namely "symbol system", "communication channel", "meaning potential". In this paper, modality is regarded as various symbol resources, including text, picture, chart, animation, color, etc. Interaction of different modalities can convey the discourse meaning, and the repeated types of discourse constitute a genre confined in the scope of the thesis defense.

\section{B. Multimodal Discourse Analysis}

Since the American linguist Harris put forward the research direction of discourse analysis in the early 1950s, Discourse analysts around the world have made great efforts in it. Various theories and methods of discourse analysis have been proposed. With the development of the times, the text constructed by literal symbols alone cannot meet the needs of meaning expression and communication. Since the 1990s, a group of researchers began to turn their attention to multimodal discourse analysis. Nowadays, the theory of multimodal discourse analysis has become a hot topic. Kress and van Leeuwen (2001) believe that multimodal discourse is a discourse that contains voice, text, image, and other communication modes to convey information. Zhu (2007) points out that multimodal discourse analysis can integrate language and other related meaning resources. It can be seen that language system and other non-verbal symbol systems such as image, music, and color together play a role in the process of meaning exchange to make the interpretation of discourse meaning more comprehensive and accurate and to help people understand how to use multimodality to achieve the purpose of social communication. Zhang (2009) believes that multimodal discourse refers to the use of auditory, visual, tactile, and other senses to communicate through language, image, sound, action, and other means and symbol resources. To sum up, we can see that multimodal discourse analysis is no longer a single analysis of language symbols, but combined with the text, music, images, animation, etc., to analyze different effects of multi-modes on the meaning of the text.

\section{Stylistics Characteristics of PowerPoint Genre}

Yates and Orlikowsi (2007) hold that genre possesses some discursive norms that are understood as entailing expectations about the following aspects of communication: purpose, content, form, participant, time, and place. More details are presented in the following table.

TAB. 1

YATES AND ORLIKOWSI'S CLASSIFICATION OF NORMS OF PPT GENRE

\begin{tabular}{|c|c|}
\hline Norms of genre & Detailed description \\
\hline purpose & $\begin{array}{l}\text { Genre always enables people to anticipate the socially recognized } \\
\text { intention. As far as PPT presentation is concerned, it is always typically } \\
\text { used to inform, explain, or persuade the audience. }\end{array}$ \\
\hline content & $\begin{array}{l}\text { Genre can make people expect the content of a presentation, and the } \\
\text { typical structure of PPT presentation can help people make or } \\
\text { understand the content of the presentation. }\end{array}$ \\
\hline form & $\begin{array}{l}\text { Genre provides the anticipation of its form, including the medium, } \\
\text { methods of construction, and linguistic elements. The standard form of } \\
\text { PPT presentation is when one person stands in front of a group of } \\
\text { people and gives a speech, while showing slides. The turn of the slide } \\
\text { depends on the progress of the presentation. }\end{array}$ \\
\hline participant & $\begin{array}{l}\text { Genre can make an expected estimate of the participants and their roles } \\
\text { involved in the interaction of information transmission. Sometimes a } \\
\text { PowerPoint presentation is done collaboratively by several people, } \\
\text { either in a way that each person is responsible for part of the } \\
\text { presentation or one person. }\end{array}$ \\
\hline time & $\begin{array}{l}\text { Genre can often be expected at specific times, although these estimates } \\
\text { are not necessarily apparent, generally in the morning, afternoon, and } \\
\text { evening, a period conducive to group and activity. }\end{array}$ \\
\hline place & $\begin{array}{l}\text { Genre can also anticipate the choice of place, but these expectations are } \\
\text { always obvious, such as in a large room or a hall with electronic } \\
\text { projection. }\end{array}$ \\
\hline
\end{tabular}

As for the categorization of PPT as a genre, Hu (2007) divided it into four types according to its different functions usually performed, namely, prompting type, visualizing type, analyzing type, and persuading type. The main function of 
the prompting type is to help the demonstrator organize thoughts with a clear hierarchy, prominent points, and orderly speech. Thus, this function is mainly realized by the modality of bullet points. Visualizing type presents ideas intuitively, by means of the modality of pictures. Analyzing type aims to explore the operating system, internal relationship, or development of something, using the modality of charts. The persuading type is employed to make the audience feel the beauty of PPT to achieve the goal of persuading, with the help of color, animation, and music modalities. It should be noted that these four types are prototypes, and combining some functions with some modalities can produce different styles.

In this paper, 7 PPTs are regarded as 7 multimodal discourses realized by multimodalities, including bullet point, text-only, text and pictures, text and chart, and animation. When these multimodal discourses are considered in the context of the thesis defense, PPTs as a whole are given meanings as a genre, embodying its conventional meanings. Hu's (2007) classification of PPT as a genre will be adopted as the theoretical framework.

\section{RESEARCH DESIGN}

\section{A. Research Data}

7 PPTs in an MA thesis defense, presented by 7 masters who majored in Foreign and Applied Linguistics are taken as the research data. Each PPT is numbered from 1 to 7 . These 7 masters are required to give a presentation to their thesis using PPT, no more than 20 minutes. There are 152 slides in the 7 PPTs. The longest one consists of 38 slides, and the shortest one includes 12 slides. The average length of all PPT presentations is about 22 slides. The content of these PPTs covers the following aspects, research background, research significance, literature review, research questions, methodology, results, discussions, findings, and limitations.

\section{B. Research Questions}

Using the theory of multimodal analysis as the theoretical basis and 7 PPTs in an MA thesis defense as the research data, this paper uses a combination of qualitative and quantitative methods to mainly answer the following questions:

1) What are the distributions and realizations of genres and modalities of PPTs in this MA Thesis Defense?

2) How is the meaning of MA thesis defense constructed multimodally?

3) What are the characteristics of PPT as a thesis defense genre?

\section{RESULTS AND DiSCUSSION}

Before presenting the research results, something vital needs to be clarified first. Guided by the categorization of PPT's functions proposed by $\mathrm{Hu}$ (2007), this paper makes some adjustments to serve this research better. Firstly, the analyzing type can be achieved not only by the modality of text and chart but also by text-only modality. Secondly, in the context of the specific research data, some slides that are difficult to be classified into any of these four types (prompting type, visualizing type, analyzing type, and persuading type) fall into the connecting type according to the author's judgment of PPT's function. Thirdly, according to Hu (2007), the persuading type can be realized using the animation modality. Given that animation modality can also be applied to the other three types, it is hard to identify its category. Thus, the author will make a careful, cautious, and precise classification of them in line with the principle of PPT's function. The classification results will be examined by another person to make sure the reliability of the classification. To be honest, these types are interacted and intertwined with each other, while they will be treated as distinct for analytic purposes.

\section{A. Overall Distribution of Types of PPT}

According to statistics, the above four types have been used in 7 PPT and 152 slides. Among all the PPTs, the overall distribution of types of PPT are as followed: it is obvious to see that the occurrence of analyzing type own the highest frequency, that is 72 , followed by connecting type, 30; prompting type, 28; persuading type, 21; and at last visualizing type, just 1 . The detailed distribution of types of PPT in each PPT is presented in Table 2. 
TABLE. 2

OVERALL DistRIBUTION OF TYPES OF PPT AS A GENRE

\begin{tabular}{|c|c|c|c|c|c|c|c|c|}
\hline type & No.1 & No. 2 & No.3 & No.4 & No.5 & No.6 & No.7 & $\begin{array}{c}\text { Total } \\
\text { number }\end{array}$ \\
\hline $\begin{array}{l}\text { Prompting } \\
\text { type }\end{array}$ & 1 & 4 & 1 & 3 & 2 & 11 & 6 & 28 \\
\hline $\begin{array}{c}\text { Visualizing } \\
\text { type }\end{array}$ & 0 & 1 & 0 & 0 & 0 & 0 & 0 & 1 \\
\hline $\begin{array}{c}\text { Analyzing } \\
\text { type }\end{array}$ & 11 & 1 & 12 & 7 & 17 & 13 & 10 & 72 \\
\hline $\begin{array}{l}\text { Persuading } \\
\text { type }\end{array}$ & 1 & 4 & 0 & 6 & 9 & 1 & 1 & 21 \\
\hline $\begin{array}{c}\text { Connecting } \\
\text { type }\end{array}$ & 2 & 2 & 2 & 2 & 10 & 6 & 6 & 30 \\
\hline $\begin{array}{c}\text { Total } \\
\text { number }\end{array}$ & 15 & 12 & 15 & 18 & 38 & 31 & 23 & 152 \\
\hline
\end{tabular}

The proportion of each kind is displayed directly and clearly in the following figure 1 :

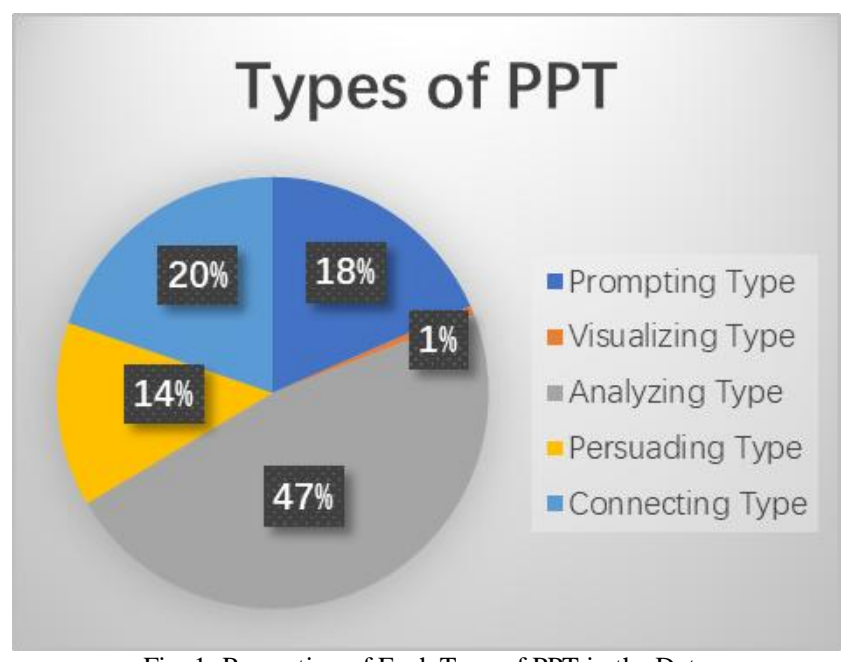

Fig. 1: Proportion of Each Type of PPT in the Data

Among all the categories, prompting type takes up $47 \%$ of the total occurrence frequency of 7 PPTs for the MA thesis defense, which is in the first place. This shows that analyzing type is commonly employed in PPTs for MA thesis defense. The occurrence frequency numbers of the connecting type and the prompting are nearly the same, which are $20 \%$ and $18 \%$ respectively, and both bear a similar function that helps the demonstrator organize thoughts clearly and orderly. The persuading type accounts for $14 \%$, whose function of persuading and inducing. While the visualizing type takes up the least proportion of these 4 types, indicating that these masters' mere willingness toward this intuitive function. Maybe the academic nature of PPT for thesis defense is the main factor that can explain the extremely low proportion. Then, each type of PPT for the MA thesis will be expounded in detail, regarding how various modalities function in these types.

\section{B. Prompting Type}

The main function of the prompting type is to help the demonstrator organize thoughts with a clear hierarchy, prominent points, and orderly speech. This type is mainly realized by using bullet point, a typographical symbol used to introduce items in a list. This type is employed by these masters to introduce the contents, research questions, research findings, and suggestions and limitations of their thesis. Some examples are presented below:

\section{Contents}

\section{Research Background}

2. Significance and Research Questions

\section{Methodology}

\section{Results}

\section{Findings and Implications}

Fig. 2: The Second Slide from No.5 PPT 


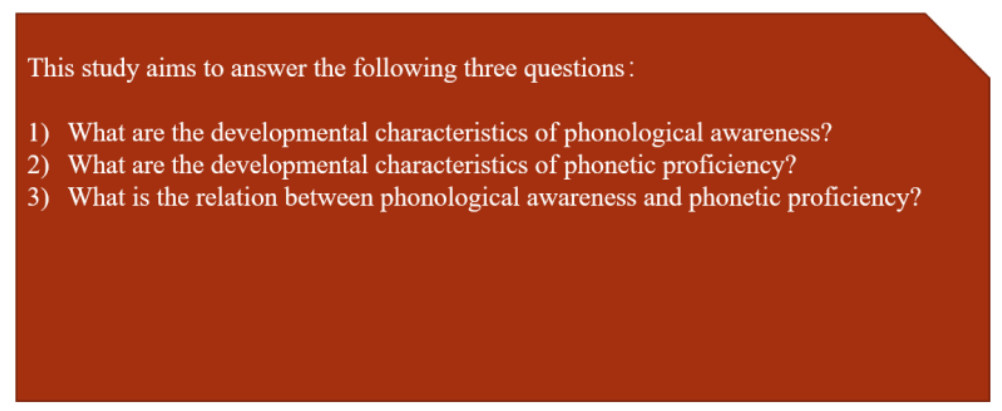

Fig. 3: The $13^{\text {th }}$ Slide from No. 6 PPT

\section{Implications}

(1) Learners with different phonetic levels showed different developmental characteristics on both phonological awareness and phonetic proficiency, teachers should try to make appropriate course design for students at different levels.

(2) The developmental characterises of PA and PP could be taken into consideration. For example, onset-rime knowledge could be put at the beginning of teaching, and syllable and phoneme knowledge, and prosodic features at a later phase.

(3) The relationship between PP and PA indicates that teachers could try to teach phonology-concerned knowledge, such as syllables, onset-rime and phonemes to help them better understand pronunciation and learn more effectively. (4) Dubbing brought learners fun; it could be used in pronunciation teaching.

\section{Limitations}

1) the length of experiment is relatively short:

2) there could be more subjects.

3) the backgrounds of each student could be considered in to analyse the factors that might be in function of their pronunciation proficiency.

Fig. 4: The $30^{\text {th }}$ Slide from No.6 PPT

The characteristics of these parts are simple and short, suitable to be displayed in the form of bullet points. When presenting the defense PPT, the principle of conciseness and indication should be abided by. Especially for the content part, as the beginning of the presentation, it should be avoided that using a whole text to explain the content of the thesis, resulting in an impression of being lengthy and boring. Figure 2 represents that this defense presentation will be given from these aspects, research background, significance and research questions, methodology, results, and findings and implications. It is explicit and clear to be listed by means of bullet pint. It is interesting that all the 7 masters use prompting type to demonstrate the content part of their theses.

The significance of the research question part cannot be overstated, being the most interesting and attractive part of the defense teachers. Research questions are the core of the whole research work and the starting point of research design, affecting all aspects of research. Listing research questions clearly and directly in the PPT is necessary and important, helping the defense teachers grasp the core of the research, and arousing their interest.

When it comes to the research finding, suggestion, and limitation part, it is impressive to see they belong to disparate types, including the prompting type, analyzing type, or persuading type, performing different functions, and it indicates demonstrators' various purposes. In this part, the defense meaning conveyed by the prompting type is chiefly concentrated on. By using the prompting type, the demonstrator intends to highlight the contributions and limitations of the thesis logically, making the defense teachers grasp the key points promptly.

Besides, the use of prompting type has a strong personal inclination. The owner of No.6 PPT prefers using prompting type to present her thoughts, while as for the demonstrators of No.1 and No.3, they rarely express their content with the help of prompting type.

All in all, using prompting type can help the demonstrator organize their thought logically and clearly, and emphasize what they most want to express, and help defense teachers get the main point of their presentation.

\section{Visualizing Type}

Visualizing type presents ideas intuitively, by means of the modality of pictures. At most times, the picture is not used casually; instead, it will be selected scrupulously to fulfill its function of intuitiveness. The relationship between the picture and the text should be closed and content-related. In this paper's data, visualizing type is only used once, which appears in the No.2 PPT. 


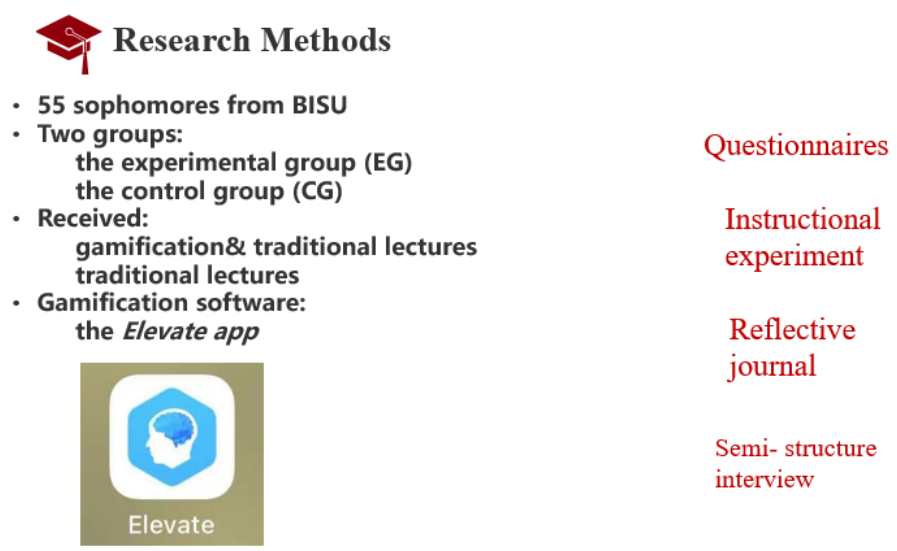

Fig. 5: The $6^{\text {th }}$ Slide from No.2 PPT

For this slide, the demonstrator is presenting her research methods, including the participant, software Elevate, and the instruments for data collection. Since Elevate will be used as the main teaching instrument in her research, occupying a core position; thus, it is out of necessity and importance to show defense teachers what this software looks like. However, it would have been better to display the operating interface to make the audience have a basic understanding of this software and deepen the impression.

On the one hand, given the seriousness and scholarship of the thesis defense, it will be inappropriate to use many pictures in the PPT presentation of the thesis defense, which is part of the reasons why this type is employed rarely. On the other hand, seriousness and scholarship should not become the obstacles to using visualizing type; realistic necessity comes first.

\section{Analyzing Type}

Analyzing type aims to explore the operating system, internal relationship, or development of something, using the modality of text only, and chart and text. Analyzing type is the most used type in the data, and so is in almost every PPT. It is widely used in academic discourse. In these 7 PPTs, analyzing type can be mainly seen in the part of research background, research design, and results and discussion.

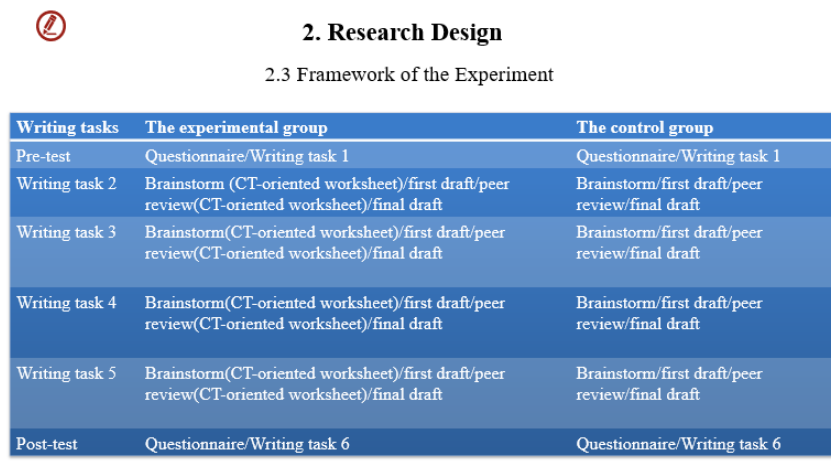

Fig. 6: The $9^{\text {th }}$ Slide from No.1 PPT

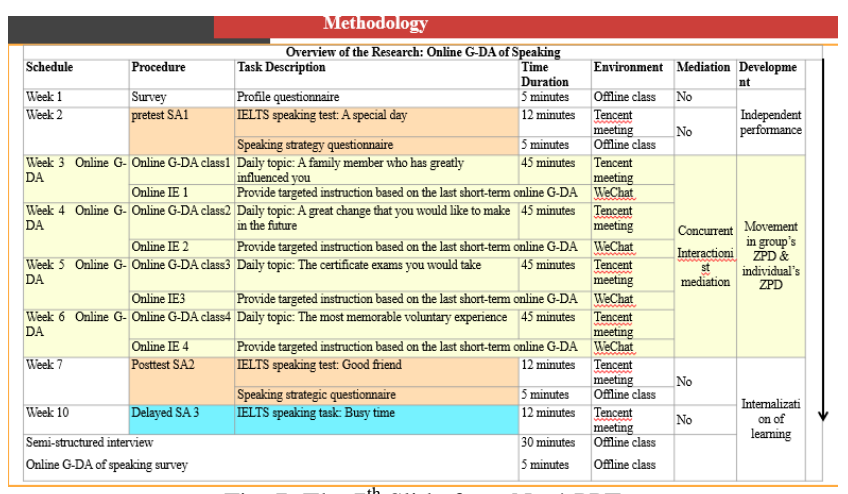

Fig. 7: The $7^{\text {th }}$ Slide from No.4 PPT 


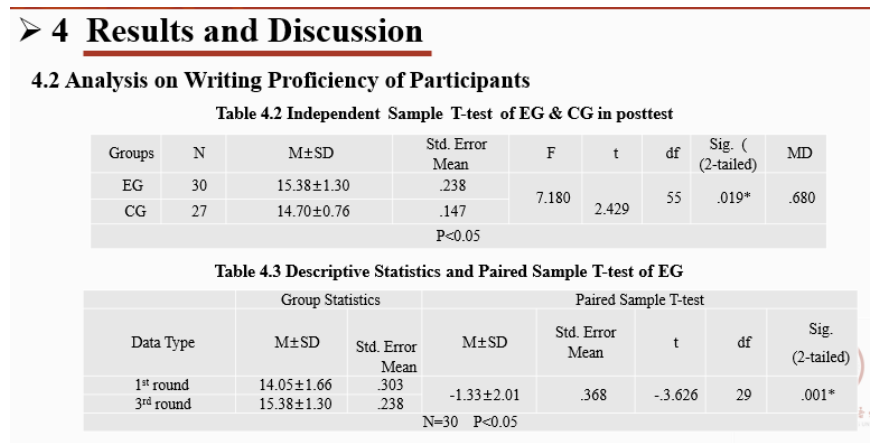

Fig. 8: The $9^{\text {th }}$ Slide from No.3 PPT

For example, figure 6 displays the framework of the research experiment, including the information of writing tasks for both the experimental group and the control group. It will be much lengthy and redundant to express the task distribution by text only. However, when the information is put into a table, it would be much explicit and straightforward, and the purpose of analyzing can be achieved by the demonstrator's speech. Figure 7 presents the overview of the research in time order, full of memorious information, such as procedures, tasks, time duration, environment, mediation and development. Figure 8 is the result part of the thesis, usually the quantitative data. Using a table to display a great deal of information is a labor-saving and visual way; however, it should be noted that there is an essential difference between the visualizing type that use picture modality as the realization method and the analyzing type that use chart modality as the realization method, that is whether a detailed and complementary illustration will be needed. It is not enough to merely put the chart on the slide; the demonstrator needs to give a comprehensive explanation and analysis of it to fulfill the function of analyzing.

(2)

1. Research Background and Significance

1.1 Research background

The critical thinking (CT) ability development of undergraduates has

been put on national agenda as illustrated in the National Standard for

Undergraduate Specialty Teaching Quality in Ordinary Institutions of

Higher Education. Writing course exerts the most significant influence on

students' critical thinking skills (Tsui, 1999).

Fig. 9: The $3^{\text {rd }}$ Slide from No.1 PPT

The analyzing type, achieved by the text modality only, can be found in the part of research background and discussion of the thesis, where the information can be difficult to be displayed by a chart. Slides with text modality only are not very common. It is common sense that putting text only in one slide without anything is tedious; thus, 7 masters try to avoid this type. The analyzing function for analyzing type is mainly achieved by text and chart modality, conveying the defense meaning that the research is professional and the analysis is both intuitive and comprehensive.

\section{E. Persuading Type}

The persuading type is employed to achieve the goal of persuading with the help of color, animation, and music modalities. In the data, the persuading function is mainly realized by color and animation modalities. This type is the most difficult one to be identified since the color and animation modalities can also be used in another 4 types. The standard of whether a slide should fall into the persuading type lies in the actual function it performs.

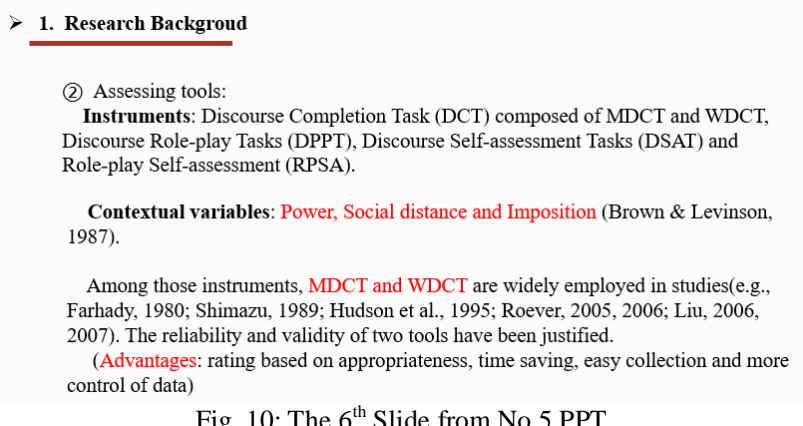

Fig. 10: The $6^{\text {th }}$ Slide from No.5 PPT 


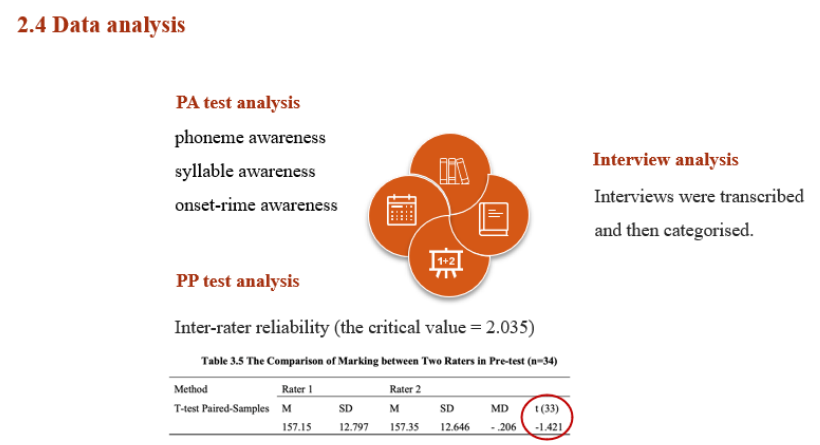

Fig. 11: The $16^{\text {th }}$ Slide from No.6 PPT

In figure 10, important information or something that the demonstrator wants to highlight is featured by red color, for example, the contents of contextual variables, assessing tools, and the advantages. By doing this, the demonstrator tries to emphasize the authority and the advantages of the assessing tool in her thesis. In figure 11, the red circle is animated, making it conspicuous in the slide. The demonstrator wants to prove that there is a balance and no significant difference between the two raters; inter-rater reliability was measured by using t-test Paired-Samples. Scores from the pre-test were used to examine the agreement. Furthermore, the results are presented in the slide. The $t$ value is circled, indicating that no significant difference between scores marked by two raters. Hence, the average score by two raters for each student in each test round can be used as the final score.

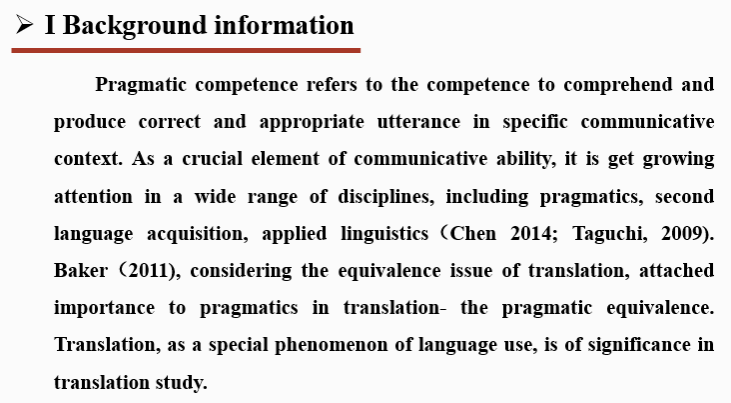

\section{Fig. 12: The $4^{\text {th }}$ Slide from No.7 PPT}

However, not all animation modalities can fulfill the function of the persuading type. Taking figure 12 as an example, although the text is animated, there exists no persuading function. There is no point in animating the whole content in one slide. Thus, this slide should be classified into the analyzing type, not the persuading type. When the persuading type of PPT is adopted, the defense meaning is focused on suggesting and persuading the defense teachers; thus, this type is usually used to convey some personal thoughts, distinct findings, and contributions of the thesis to support the demonstrator's point of view.

\section{F. Connecting Type}

In the context of the research data, some slides that are difficult to be classified into any of these four types (prompting type, visualizing type, analyzing type, and persuading type) fall into connecting type. The connecting type is employed to undertake a simple summary of the analysis above or lead to the following part. Both the first introducing slide and the last acknowledgment slide belong to the type.

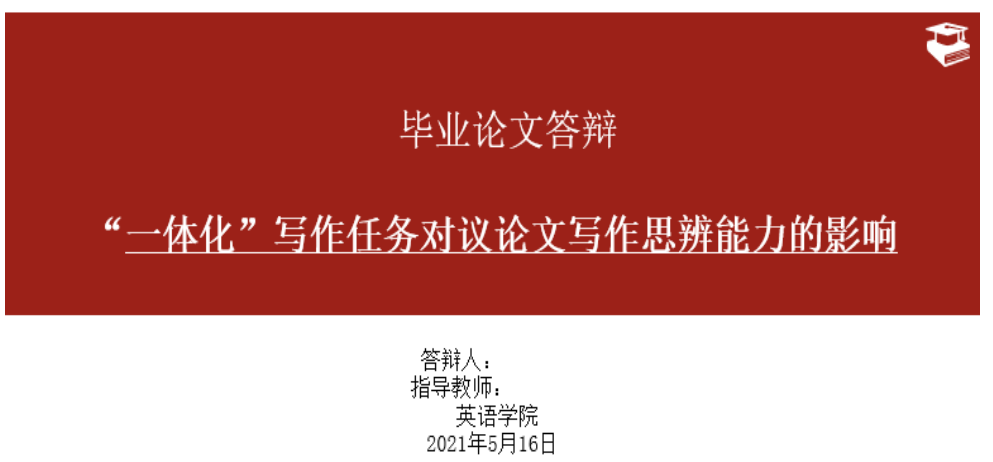

Fig. 13: The $1^{\text {st }}$ Slide from No.1 PPT 


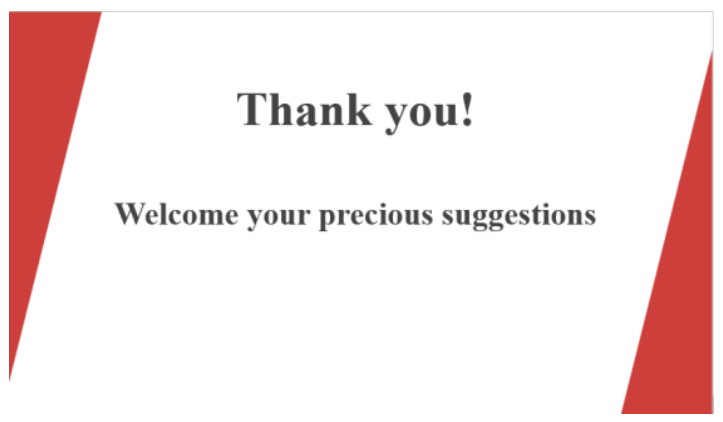

Fig. 14: The $18^{\text {th }}$ Slide from No.4 PPT

\section{Methodology}

Fig. 15: The $8^{\text {th }}$ Slide from No.7 PPT

It is a type that can indicate the individual usage tendency. As common sense, the first four PPTs only adopt the connecting type in the beginning and at the end of their presentations, while the last 3 PPTs use the connecting type with a high frequency. It is hard to give a conclusion which choice is better. On the one hand, the connecting type can give the audience a response time, and get ready to welcome the next information. On the other hand, an over-used this type can make the PPT seem loose and incoherent. Thus, Proper use is the key.

\section{CONCLUSION}

According to the results and discussions above, it can be seen that five types of PPT are all adopted in the thesis defense, with different occurrence frequencies. The main purpose of a thesis defense is to explain and interpret it; thus, the analyzing function is the primary concern. The use of connecting, persuading, and prompting type has a strong personal tendency, varying from person to person. However, the demonstrator's purpose should be the principle and standard of choosing. Besides, the misuse of the persuading type should be taken notice of, avoiding being counterproductive. The visualizing type takes up the lowest proportion among them, indicating that pictures are the last modality that demonstrators tend to use, making sure the PPT defense presentation is academic and serious. All in all, the demonstrators need to know different types of PPT, the characteristics, and modalities that can achieve different functions in order to present the thesis in an effective and appropriate way.

Based on the analysis and discussion above, the characteristics of PPT as a thesis defense genre can be summarized as followed:

First, the purpose of PPT as a thesis defense genre is to present the respondent's thesis comprehensively and logically, explaining to and persuading the defense teachers the worth of the respondent's thesis.

Second, the content of PPT as a thesis defense genre typically contains research background, research significance, literature review, research questions, methodology, results, discussions, findings, and limitations of the thesis, with an introducing slide and a acknowledge slide at the beginning and the end of PPT respectively. You do not have to deal with all the elements above, while some core information has to be included, such as research questions, methodology, research results, and research findings.

Third, the standard form of PPT as a thesis defense genre is when one respondent stands in front of a group of defense teachers and gives a speech to their theses while showing slides. In most cases, manual operation of the turn of the slide is adopted, which is convenient to control and adjust the time and pace of the presentation.

At last, according to reality, time and space vary among different schools. At most times, the place is in a large room or hall with electronic projection. However, because of the prevalence of COVID-19, online defense is gradually accepted.

\section{REFERENCES}

[1] Altman, R. (2004). The Deadly Sins of Modern PowerPoint Usage. Online Corporation. (accessed 25/6/2021). 
[2] Gu Ping. (2017). The construction of pragmatic identity in the defense of master's thesis for English majors. Master dissertation, Xi'an Technological University.

[3] Gu Yueguo. (2007). Analysis of multimedia and multimodal learning. Technology Enhanced Foreign Languages, (02), 3-12.

[4] Hu Zhuanglin, (2007). PowerPoint: Tool, Text, Genre, and Style. Foreign Language Education, 28(4), 4-5.

[5] Ke Xianbing. (2010). Research on defense response strategies based on the types of questions in defense conversation. Journal of Xianning College, (04), 93-97.

[6] Knoblauch, H. (2012). PowerPoint, communication, and the knowledge society. London: Cambridge University Press.

[7] Kress, G., \& Van Leeuwen, T. (2001). Multimodal discourse. The modes and media of contemporary communication. (Cappelen, London 2001).

[8] Li Na, \& Jing Xiaoping. (2019). The construction of respondent's pragmatic identity in academic interaction. Journal of Zhejiang International Studies University, (01), 41-47.

[9] Recski, L. (2005). Interpersonal engagement in academic spoken discourse: A functional account of dissertation defenses. English for Specific Purposes, 24(1), 5-23.

[10] Simons, T. (2005). Does PowerPoint make you stupid. Presentations, 1(8), 3.

[11] Tuffe Edward, R. (2003). PowerPoint is Evil. Weird Minizine. (accessed 25/6/2021).

[12] Yates, J., \& Orlikowski, W. (2007). Communicative practices in workplaces and the professions: Cultural perspectives on the regulation of discourse and organizations. IN Yates, J., \& Orlikowski, W. (Eds.), The PowerPoint presentation and its corollaries: How genres shape communicative action in organizations. New York: Routledge, 67-92.

[13] Zhang Delu. (2009). Application of multimodal Discourse Theory and media technology in foreign language teaching. Foreign Language Education, (04), 15-20.

[14] Zhou Xinnian, Zhang Zhenxiong, \& Qiu Rongzu. (2008). Process and skills of master's thesis defense. Chinese Forestry Education, (03), 44-46.

[15] Zhu Yongsheng. (2007). Theoretical basis and research methods of multimodal discourse analysis. Foreign Language Research, (05), 82-86.

Zhencong Liu is currently Associate Professor in the School of English Language, Culture, and Literature, Beijing International Studies University, China. He received his PH.D degree in linguistics from Beijing Foreign Studies University, China in 2007. His research interests include general linguistics, cognitive linguistics, language teaching and research. Dr. Liu has published more than 11 textbooks and 30 research papers on cognitive and applied linguistics.

Hui Liu (corresponding author) was born in Hebei province, China in 1997. She will receive her Master Degree from Beijing International Studies University, China in 2023. Her research interests include second language acquisition, corpus linguistic and English teaching, and discourse analysis. 